\section{Tracing the Origin, Spread, and Molecular Evolution of Zika Virus in Puerto Rico, 2016-2017}

\author{
Gilberto A. Santiago, ${ }^{1}$ Chaney C. Kalinich, ${ }^{1}$ \\ Fabiola Cruz-López, Glenda L. González, \\ Betzabel Flores, Aaron Hentoff, Keyla N. Charriez, \\ Joseph R. Fauver, Laura E. Adams, Tyler M. Sharp, \\ Allison Black, Trevor Bedford, Esther Ellis, Brett Ellis, \\ Steve H. Waterman, Gabriela Paz-Bailey, \\ Nathan D. Grubaugh, ${ }^{2}$ Jorge L. Muñoz-Jordán²
}

Author affiliations: Centers for Disease Control and Prevention, San Juan, Puerto Rico, USA (G.A. Santiago, F. Cruz-López, G.L. González, B. Flores, K.N. Charriez, L.E. Adams, T.M. Sharp, G. Paz-Bailey, J.L. Muñoz-Jordán); Yale School of Public Health, New Haven, Connecticut, USA (C.C. Kalinich, A. Hentoff, J.R. Fauver, N.D. Grubaugh); US Public Health Service, Rockville, Maryland, USA (L.E. Adams, T.M. Sharp);

Fred Hutchinson Cancer Research Center, Seattle, Washington, USA (A. Black, T. Bedford); US Virgin Islands Department of Health, Charlotte Amalie, St. Thomas, Virgin Islands, USA (E. Ellis, B. Ellis)

\section{DOI: https://doi.org/10.3201/eid2711.211575}

We reconstructed the 2016-2017 Zika virus epidemic in Puerto Rico by using complete genomes to uncover the epidemic's origin, spread, and evolutionary dynamics. Our study revealed that the epidemic was propelled by multiple introductions that spread across the island, intricate evolutionary patterns, and $\approx 10$ months of cryptic transmission.

P uerto Rico reported the first confirmed case of Zika virus (ZIKV) disease in November 2015 and subsequently experienced epidemic transmission that peaked by mid-August 2016 (1). Despite the large number of confirmed cases detected by traditional surveillance, the origin, spread, and evolutionary dynamics of this epidemic remain undetermined. We sought to reconstruct the epidemic transmission period by using a genomic epidemiology approach and determine evolution of the virus in the island.

To investigate the emergence and subsequent epidemic of ZIKV in Puerto Rico, we generated 83 complete genomes $(2,3)$ directly from PCR-positive serum samples (4) (Appendix, https://wwwnc.cdc. gov/EID/article/27/11/21-1575-App1.pdf) collected

${ }^{1}$ These first authors contributed equally to this article.

${ }^{2}$ These senior authors contributed equally to this article. from the 8 health regions of Puerto Rico during March 2016-January 2017, congruent to a geotemporal representation of the epidemic in the island. We then performed phylogenetic analysis with an additional 233 published genomes from GenBank that represent the emergence and spread of ZIKV in the Americas during 2015-2017. The resulting reconstructed phylogeny was consistent with published tree topologies, nucleotide substitution rate ranges, and divergence patterns observed elsewhere for the entirety of the Americas (Appendix Figure 1, panel A), providing a pragmatic context to the proposed model of spread and divergence of ZIKV in Puerto Rico (5). At least 8 separate foreign-introduction events were captured within the ancestry of the viruses sequenced, including 2 that expanded into autochthonous lineages and 6 separate introduction events represented by individual sequences associated with genomes from the United States, the Caribbean, South America, and Central America, thus suggesting limited spread.

In addition, we analyzed the temporal molecular evolutionary signal in our dataset by reconstructing time-calibrated phylogenies by using genomes annotated with date of sample collection based on year, month, and days for temporal precision. The correlation between date of sample collection and root-totip genetic distance supported the heterochronous nature of our dataset. The estimated divergence from the root (i.e., time of most recent common ancestor [tMRCA] of this tree) occurred in February 2013 (because 2013-2014 ZIKV genomes from French Polynesia were used as the root), and the within-epidemic evolutionary rate was $1.09 \times 10^{-3}$ substitutions/site/ year (Appendix Figure 1, panel B).

Bayesian reconstruction of Puerto Rico clade 1 (PR C1) presents the largest autochthonous monophyletic cluster that originated from viruses from South America and the Caribbean, including Brazil, Suriname, French Guyana, the US Virgin Islands, and Dominican Republic (Figure). tMRCA estimates place the divergence of PR C1 in mid-June 2015 (95\% highest posterior density [HPD] February 2015-October 2015) and a within-outbreak evolutionary rate of $1.61 \times 10^{-3}$ (95\% HPD 1.13-2.10 × 10-3) substitutions/site/year. In addition, PR C1 was observed to diverge further into 2 subclades (SC1 and SC2) spreading across the island. The second clade, Puerto Rico clade 2 (PR C2), presents a smaller autochthonous monophyletic cluster that originated from viruses in Central America, including Nicaragua and Honduras (Figure). Our tMRCA estimates placed the emergence of PR C2 in February 2016 (95\% HPD October 2015-April 2016) and its evolutionary rate was similar to PR C1 at $1.87 \times 10^{-3}$ 
(95\% HPD 1.1-2.64 × 10-3). We compared the ZIKV epidemic history of Puerto Rico to the time-calibrated Bayesian phylogenies and observed that the tMRCA of PR C1 precedes the initial confirmation of ZIKV in

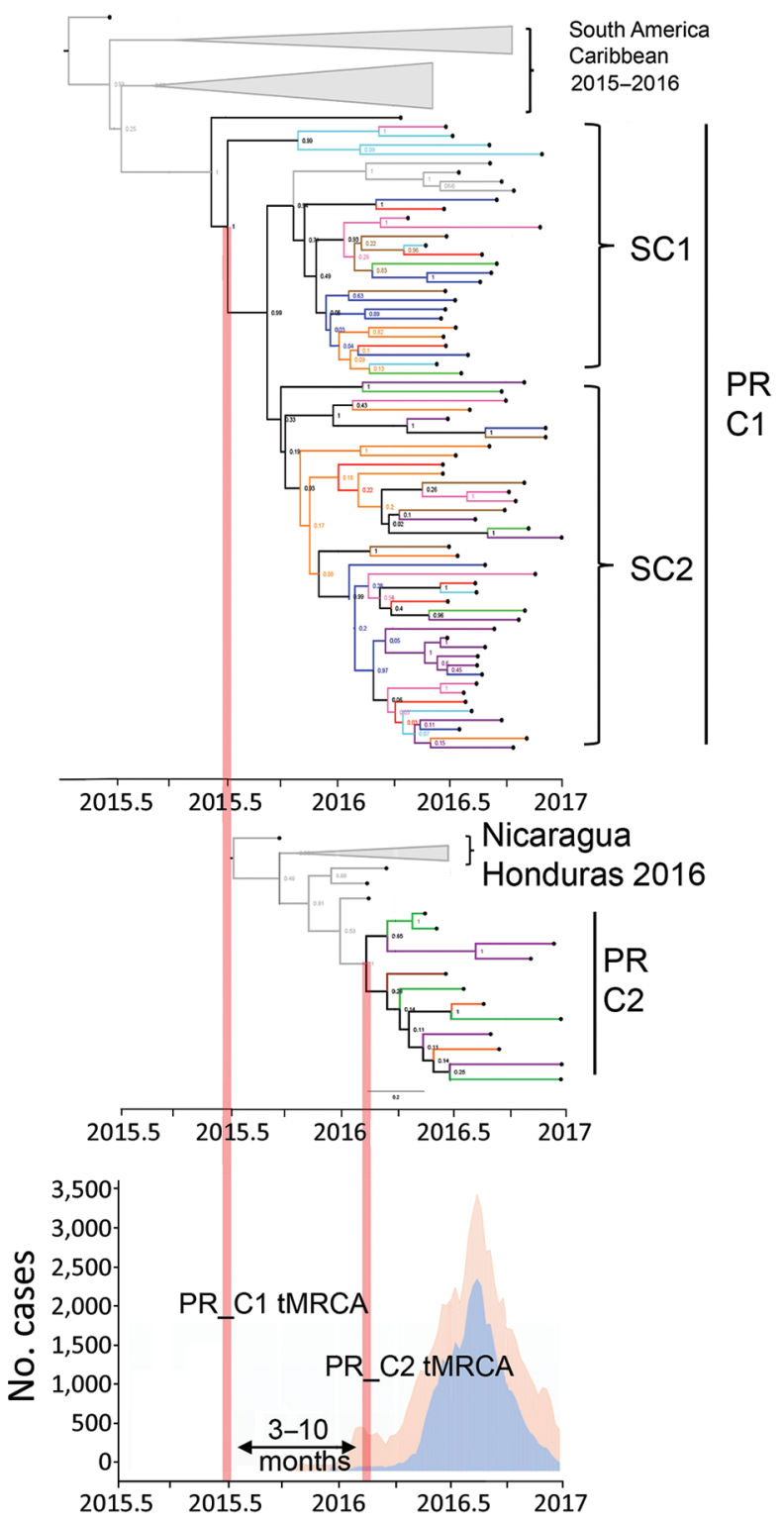

Figure. Intra-island spread and divergence of Zika virus, Puerto Rico, 2016-2017. Bayesian phylogenetic reconstruction using maximum clade credibility trees shows genomes grouping with 2 separate clusters. PR C1 is associated with genomes from South America and the Caribbean (top); this clade diverged into SC1 and SC2. PR C2 is associated with genomes from Central America (center). Epidemic curve of total Zika cases per week (orange shade) and cases confirmed by reverse transcription PCR per week (blue shade) during 2015-2017 (bottom). All external branches representing Puerto Rico genomes are color-coded according to the 8 health regions of Puerto Rico: region 1, red; region 2, blue; region 3 , orange; region 4, green; region 5 , purple; region 6 , cyan; region 7, brown; and region 8, magenta. C, clade; PR, Puerto Rico; SC, subclade; tMRCA, time of most recent common ancestor. the island through traditional surveillance methods by 3-10 months and that expansion of all PR lineages coincides with the peak of the epidemic curve (Figure). We assessed phylogenetic clustering patterns for geographic association with each of the health regions and detected none (Appendix Figure 2).

We inferred past viral population dynamics by using Bayesian Skygrid plots, which show an increase in genomic diversity that coincides in time with the emergence of ZIKV in the Americas, followed by a series of fluctuations in the effective population size, characteristic of the virus spreading rapidly through the region (Appendix Figure 3). In Puerto Rico, we observed a similar sharp increase upon emergence and subsequent patterns that mirror the trends observed in the Americas.

Our study revealed the origin and epidemic spread of ZIKV in the island after a period of cryptic transmission undetected by traditional surveillance. Similar cryptic transmission was reported in Brazil and Colombia (6-8), where case detection was hindered by the difficulty to capture asymptomatic or mild cases with clinical manifestations that overlap endemic arboviruses and other laboratory testing limitations particular to ZIKV (9). The dataset we generated in our study presents a relevant contribution to the geotemporal sampling of ZIKV genomes from the region, enabling the study the evolutionary and epidemic dynamics in the Americas.

The integration of genomic epidemiology to arbovirus surveillance has proven to be central to the ascertainment of disease epidemiology, uncovering information otherwise concealed by the nature of the disease and limitations of surveillance systems. Fundamentally, integrated proactive genomic surveillance may help us to predict virus emergence and mitigate more effectively their regional or global expansion.

\section{Acknowledgments}

We thank the collaborators from the Ponce Medical School Foundation, Inc. (grant no. U01CK000580), the Puerto Rico Health Department, and members of the Puerto Rico Zika Task Force for the valuable contributions to the enhanced surveillance during the Zika outbreak in 2016.

This project was partially funded by the Centers for Disease Control and Prevention's Advanced Molecular Detection Program and the Yale University's School of Public Health start-up package provided to N.D.G. Additional support for coauthors C.K. and A.H. was provided by the Yale University's Jackson Institute of Global Health Field Experience Award and the Yale Collaborative Action Fellowship. 


\section{About the Author}

Dr. Santiago is a lead research microbiologist at the Centers for Disease Control and Prevention in San Juan, Puerto Rico. His research is focused on the development of molecular diagnostic tests and genomic epidemiology of dengue virus and severe acute respiratory syndrome coronavirus 2 .

\section{References}

1. Sharp TM, Quandelacy TM, Adams LE, Aponte JT, Lozier MJ, Ryff K, et al. Epidemiologic and spatiotemporal trends of Zika virus disease during the 2016 epidemic in Puerto Rico. PLoS Negl Trop Dis. 2020;14:e0008532. https://doi.org/10.1371/journal.pntd.0008532

2. Quick J, Grubaugh ND, Pullan ST, Claro IM, Smith AD, Gangavarapu K, et al. Multiplex PCR method for MinION and Illumina sequencing of Zika and other virus genomes directly from clinical samples. Nat Protoc. 2017;12:1261-76. https:/ / doi.org/10.1038/nprot.2017.066

3. Grubaugh ND, Gangavarapu K, Quick J, Matteson NL, De Jesus JG, Main BJ, et al. An amplicon-based sequencing framework for accurately measuring intrahost virus diversity using PrimalSeq and iVar. Genome Biol. 2019;20:8. https://doi.org/10.1186/s13059-018-1618-7

4. Santiago GA, Vázquez J, Courtney S, Matías KY, Andersen LE, Colón C, et al. Performance of the Trioplex real-time RT-PCR assay for detection of Zika, dengue, and chikungunya viruses. Nat Commun. 2018;9:1391. https:/ / doi.org/10.1038/s41467-018-03772-1

5. Metsky HC, Matranga CB, Wohl S, Schaffner SF, Freije CA, Winnicki SM, et al. Zika virus evolution and spread in the Americas. Nature. 2017;546:411-5. https://doi.org/10.1038/ nature22402

6. Faria NR, Quick J, Claro IM, Thézé J, de Jesus JG, Giovanetti M, et al. Establishment and cryptic transmission of Zika virus in Brazil and the Americas. Nature. 2017;546:406-10. https://doi.org/10.1038/nature22401

7. Black A, Moncla LH, Laiton-Donato K, Potter B, Pardo L, Rico A, et al. Genomic epidemiology supports multiple introductions and cryptic transmission of Zika virus in Colombia. BMC Infect Dis. 2019;19:963. https:/ / doi.org/ 10.1186/s12879-019-4566-2

8. Grubaugh ND, Saraf S, Gangavarapu K, Watts A, Tan AL, Oidtman RJ, et al.; GeoSentinel Surveillance Network. Travel surveillance and genomics uncover a hidden Zika outbreak during the waning epidemic. Cell. 2019;178:1057-1071.e11. https:/ / doi.org/10.1016/j.cell.2019.07.018

9. Peters R, Stevenson M. Zika virus diagnosis: challenges and solutions. Clin Microbiol Infect. 2019;25:142-6. https://doi.org/10.1016/j.cmi.2018.12.002

Address for correspondence: Jorge L. Muñoz-Jordan, Centers for Disease Control and Prevention, 1324 Cañada St, San Juan, PR 00920, USA; email jmunoz@cdc.gov.

\section{Fatal Systemic Capillary Leak Syndrome after SARS-CoV-2 Vaccination in Patient with Multiple Myeloma}

\author{
Gwang-Jun Choi, Seon Ha Baek, Junmo Kim, \\ Jung Ho Kim, Geun-Yong Kwon, Dong Keun Kim, \\ Yeon Haw Jung, Sejoong Kim
}

Author affiliations: Daegu Metropolitan Government, Daegu, South Korea (G.-J. Choi); Hallym University Dongtan Sacred Heart Hospital, Hwaseong, South Korea (S.H. Baek); National Forensic Service Daegu Institute, Daegu (J. Kim); Yeungnam University College of Medicine, Daegu (J.H. Kim); Korea Disease Control and Prevention Agency, Cheongju, South Korea (G.-Y. Kwon, D.K. Kim, Y.H. Jung); Seoul National University Bundang Hospital, Seongnam, South Korea (S. Kim)

DOI: https://doi.org/10.3201/eid2711.211723

A young man with smoldering multiple myeloma died of hypotensive shock 2.5 days after severe acute respiratory syndrome coronavirus 2 vaccination. Clinical findings suggested systemic capillary leak syndrome (SCLS); the patient had experienced a previous suspected flare episode. History of SCLS may indicate higher risk for SCLS after receiving this vaccine.

Systemic capillary leak syndrome (SCLS) is an extremely rare disease of unknown incidence (1). Typical manifestations of SCLS include hypotension, edema, hemoconcentration, and hypoalbuminemia after nonspecific prodromal illnesses $(1,2)$. Increased capillary vascular permeability is the commonly accepted pathophysiology $(1,2)$. However, the exact pathogenesis remains unclear.

As part of the efforts to combat the ongoing pandemic of coronavirus disease (COVID-19), caused by severe acute respiratory syndrome coronavirus 2 , the US Food and Drug Administration on February 27, 2021, gave emergency use authorization to the Ad26.COV2.S vaccine (Johnson \& Johnson/Janssen, https:/ / www.jnj. com). An SCLS case series reported 1 case of SCLS in a patient who received the Ad26.COV2.S vaccine (3). The European Medicines Agency reviewed 3 cases of SCLS in Ad26.COV2.S vaccine recipients and issued a report, published July 9, 2021, advising against administering the vaccine in persons with previous SCLS experiences (4). We describe a case of SCLS after Ad26.COV2.S vaccination in a patient with smoldering multiple myeloma.

A 38-year-old man reporting vomiting and dizziness sought treatment at an emergency department. 